\title{
CXC and CC Chemokine Receptors on Coronary and Brain Endothelia
}

\author{
Omri Berger, ${ }^{1,8}$ Xiaohu Gan,, ,8 Chandrasekhar Gujuluva, ${ }^{1,8}$ \\ Alan R. Burns, ${ }^{4}$ Girija Sulur, ${ }^{3}$ Monique Stins, ${ }^{5}$ Dennis Way, ${ }^{6}$ \\ Marlys Witte, ${ }^{6}$ Martin Weinand, ${ }^{6}$ Jonathan Said, ${ }^{3}$ \\ Kwang-Sik Kim, ${ }^{5}$ Dennis Taub, ${ }^{7}$ Michael C. Graves, ${ }^{1}$ and \\ Milan Fiala ${ }^{8}$
}

Departments of ${ }^{1}$ Neurology, ${ }^{2}$ Microbiology and Immunology, and ${ }^{3}$ Pathology, UCLA School of Medicine, Los Angeles, California, U.S.A.

${ }^{4}$ Department of Medicine, Section of Cardiovascular Sciences and Leukocyte Biology, Baylor College of Medicine, Houston, Texas, U.S.A.

${ }^{5}$ Division of Infectious Diseases, Children's Hospital of Los Angeles, Los Angeles, California, U.S.A.

${ }^{6}$ Department of Surgery, University of Arizona, Tucson, Arizona, U.S.A.

${ }^{7}$ National Institute on Aging, Baltimore, Maryland, U.S.A.

${ }^{8}$ Department of Medicine, West Los Angeles VA Medical Center, Los Angeles, California, U.S.A.

Accepted October 25, 1999.

\begin{abstract}
Background: Chemokine receptors on leukocytes play a key role in inflammation and HIV-1 infection. Chemokine receptors on endothelia may serve an important role in HIV-1 tissue invasion and angiogenesis.

Materials and Methods: The expression of chemokine receptors in human brain microvascular endothelial cells (BMVEC) and coronary artery endothelial cells (CAEC) in vitro and cryostat sections of the heart tissue was determined by light and confocal microscopy and flow cytometry with monoclonal antibodies. Chemotaxis of endothelia by CC chemokines was evaluated in a transmigration assay.

Results: In BMVEC, the chemokine receptors CCR3 and CXCR4 showed the strongest expression. CXCR4 was localized by confocal microscopy to both the cytoplasm and the plasma membrane of BMVEC. In CAEC, CXCR4 demonstrated a strong expression with predominantly periplasmic localization. CCR5 expression was detected both in BMVEC and CAEC but at a lower level. Human umbilical cord endothelial cells (HUVEC) expressed
\end{abstract}

strongly CXCR4 but only weakly CCR3 and CCR5. Two additional CC chemokines, CCR2A and CCR4, were detected in BMVEC and CAEC by immunostaining. Immunocytochemistry of the heart tissues with monoclonal antibodies revealed a high expression of CXCR4 and CCR2A and a low expression of CCR3 and CCR5 on coronary vessel endothelia. Coronary endothelia showed in vitro a strong chemotactic response to the CC chemokines RANTES, MIP- $1 \alpha$, and MIP- $1 \beta$.

Conclusions: The endothelia isolated from the brain display strongly both the CCR3 and CXCR4 HIV-1 coreceptors, whereas the coronary endothelia express strongly only the CXCR4 coreceptor. CCR5 is expressed at a lower level in both endothelia. The differential display of CCR3 on the brain and coronary endothelia could be significant with respect to the differential susceptibility of the heart and the brain to HIV-1 invasion. In addition, CCR2A is strongly expressed in the heart endothelium. All of the above chemokine receptors could play a role in endothelial migration and repair. 


\section{Introduction}

Chemokines (up to 50) are members of the chemokine superfamily that were initially identified as chemotactic, low-molecular-weight peptides. Chemokines are classified into CXC (or $\alpha$ ), CC (or $\beta), C$, and CXXXC families (1). Although CXC chemokines with the ELR motif serve as chemoattractants for neutrophils and CC chemokines attract primarily monocytes and $\mathrm{T}$ cells, chemokine distinction based on leukocyte specificity has become blurred (2). Chemokine receptors belong to the large family of $G$ protein-coupled, seven-transmembrane domain (also called serpentine) receptors (3), which are displayed on many different cell types, including endothelia (2). Chemokines orchestrate monocyte and lymphocyte migration across the endothelial lining of blood vessels, a process that plays a central role in pathological states such as atherosclerosis (4), Alzheimer's disease (5), multiple sclerosis (6), and HIV-1 encephalitis (7). For example, monocyte chemotactic protein-1 (MCP-1) is a pivotal chemokine for monocyte migration in atherosclerosis (8), whereas in multiple sclerosis the chemokines interferon- $\gamma$ (IFN- $\gamma)$-inducible protein (IP-10), monokine induced by IFN- $\gamma$ (MIG), and regulated on activation, normal $\mathrm{T}$ cell expressed and secreted (RANTES) are considered critical for mononuclear infiltration (9). Different chemokines induce the accumulation of macrophages in fatty streaks of atherosclerosis (10), migration of monocytes across coronary endothelia in coronary atherosclerosis $(11,12)$, and monocyte infiltration of the brain in AIIS encephalitis (13).

In contrast to the detailed knowledge of the multiple effects of chemokines on leukocyte migration and signaling through chemokine receptors (14), the role of chemokine receptors on endothelia is just beginning to be unraveled. The existence of chemokine receptors on endothelia has been obscured by the presence of chemokine binding sites that do not display the chemokine subclass specificities (15) and may represent proteoglycans $(16,17)$. Specific chemokine binding sites on human brain microvessels were visualized using a biotinylated chemokine binding assay (18). Recently, the presence of CXCR4 on human umbilical cord endothelial cells (HUVEC) was shown using Northern blotting, immunocy-

Address correspondence and reprint requests to: Dr. Milan Fiala, Department of Neurology, UCLA School of Medicine, 710 Westwood Plaza, Los Angeles, CA 90095-1769, U.S.A. Phone: 310-206-6392; Fax: 310-794-5094; E-mail: fiala@ucla.edu tochemistry, and in situ hybridization (19-21). Some of these studies also suggest endothelial expression of other chemokine receptors, such as CCR3, CCR1, CCR2, and CXCR1 (20), and CCR1, CCR2, and CCR5 (18).

Chemokines and chemokine receptors play an important role in normal development and inflammatory and angiogenic responses of endothelia. In the development of the vascular system, CXCR4, with its cognate ligand stromal cell-derived factor-1 (SDF-1), is essential for vascularization of the gastrointestinal tract (22). CXCR4 is also involved in B cell lymphopoiesis, bone marrow myelopoiesis, and cardiac ventricular septum formation (23). CXC chemokines with the ELR motif, but not RANTES or MCP-1, have a potent chemotactic activity on bovine adrenal gland endothelia in vitro and in vivo, although the binding sites on endothelia have not been determined (24). Endothelial CXCR4 responds to its ligand, SDF-1, by mobilization of intracellular calcium and endothelial cell migration $(20,19)$.

Endothelial cells from different sites show significant heterogeneity in antigenic properties (25), function (26), and morphology (27). The data presented by Gupta et al. (20) and this communication suggest that endothelia may also differ in chemokine receptor display. Since the discovery in HUVEC of a predominant message for CXCR4, there has been speculation about the role of endothelial chemokine receptors and their modulation by viral proteins and cytokines in HIV-1 invasion into tissues $(20,28)$. CXCR4 transcription in endothelia is modulated by several cytokines, including IFN- $\gamma$, interleukin- $1 \beta$ (IL-1 $\beta$ ), and tumor necrosis factor- $\alpha$ (TNF- $\alpha$ ) which are elevated in AIDS $(20,21)$. Furthermore, the viral protein Tat induces dramatic upregulation of CXCR4 and CCR5 in monocyte/ macrophages, which transmigrate across the blood-brain barrier in HIV-1 encephalitis (29). The expression of the HIV-1 chemokine coreceptors on endothelia could be crucial for viral invasion into the brain that may occur either through paracellular viral transport $(30,31)$ or by a transcellular route (C. Gujuluva et al., unpublished results). Furthermore, we speculate that the receptors could be involved in the regulation of monocyte/macrophage transmigration across the blood-brain barrier and the blood-brain barrier permeability.

In this work, we have investigated the presence of CC and CXC chemokine receptors in cultured endothelia derived from the coronary 
vessels and brain microvessels, and in coronary endothelia in the heart tissues. Both coronary artery endothelial cells (CAEC) and brain microvascular endothelial cells (BMVEC) display strongly CXCR4, as described by others, but BMVEC differ from CAEC in a strong expression of CCR3. CCR5 is expressed at a lower level in both endothelia. As shown by confocal microscopy, CCR5 and CXCR4 are localized both on the plasma membrane and in the cytoplasm but the cytoplasmic localization is stronger in BMVEC than in CAEC. CAEC migrate in response to the CC chemokines RANTES, macrophage inflammatory protein- $1 \alpha(\mathrm{MIP}-1 \alpha)$, and macrophage inflammatory protein-1 $\beta$ (MIP-1 $\beta$ ).

\section{Materials and Methods}

Cells

CAEC (Clonetics/BioWhittaker, San Diego, CA) were isolated by the producer from normal human heart tissues by dissection of coronary arteries and enzymatic digestion and were propagated in $75 \mathrm{~cm}^{2}$ T.C. flasks (Falcon Plastics/ Becton Dickinson Labware, Franklin Lakes, NJ) using $E M^{R}-2-M V$ Bullet Kit medium (Clonetics/BioWhittaker).

Adult BMVEC were prepared from surgical brain tissues obtained during removal of epileptogenic foci that were dissociated and cultured by the techniques described previously (32). The outgrowing cells were purified by fluorescenceactivated cell sorting (FACS) using uptake of acetylated low-density lipoprotein labeled with 1, 1'-dioctadecyl-3,3,3', 3'tetramethylindocarbocyanine perchlorate (DiI-Ac-LDL) (Biomedical Technologies, Stoughton, MA) as described previously (32). BMVEC were propagated in $75 \mathrm{~cm}^{2}$ T.C. flasks using RPMI 1640 medium (Irvine Scientific, Santa Ana, CA) with $10 \%$ fetal bovine serum (FBS), 10\% NuSerum (Collaborative Research, Bedford, MA), sodium pyruvate, and modified Eagle's medium (MEM) nonessential amino acids and vitamins. Three different strains (two from adult brain and one from a pediatric brain) were examined for chemokine receptors.

HUVEC were prepared by harvesting umbilical veins by collagenase perfusion (33), and were pooled and plated in $75 \mathrm{~cm}^{2}$ T.C. flasks pretreated with $0.2 \%$ gelatin (Difco, Detroit, MI). HUVEC were cultured in a 1:1 mixture of M199 (Gibco BRL) and astrocyte-conditioned medium was supplemented with $10 \%$ FBS and $10 \%$ bovine calf serum (BCS) (Hyclone Labora- tories), $1 \%$ penicillin-streptomycin (Gibco BRL), $1 \%$ fungizone (Gibco BRL), 1\% HEPES buffer (Gibco BRL), $1 \mathrm{mg} / \mathrm{ml}$ heparin (Sigma), and 50 $\mathrm{mg} / \mathrm{ml}$ endothelial cell growth supplement (Collaborative Biomedical Products, Bedford, MA) (34).

The endothelia were removed from flasks using $0.05 \%$ trypsin/5 mM EDTA (Gibco BRL), seeded onto glutaraldehyde-cross-linked, gelatin-coated coverslips (34) in 24-well plates, and were grown to confluence in the medium appropriate for each cell strain. CAEC, BMVEC, and HUVEC were stained $100 \%$ positively with the antibody to von Willebrand factor and vimentin and were unstained with antibody to $\alpha$-smooth muscle actin (all from DAKO, Carpinteria, CA).

\section{Antibodies}

Monoclonal antibody (MAb) anti-CXCR4 (clones 44708.111, 44716.111, 44717.111) (R6D Systems), MAb anti-CCR3 (7B1 1) (LeukoSite), MAb anti-CCR5 (clones, 45502.111，45523.111, 45549.111, and 45531.111) (RED Systems), MAb anti-CXCR1 (also designated as IL-8RA), and MAb anti-CXCR2 (also designated IL-8RB) were from Pharmingen International (La Jolla, $\mathrm{CA})$. Goat polyclonal antibodies (PAb), antiCCR1, anti-CCR2A, anti-CCR2B, anti-CCR3, anti-CCR4, anti-CCR5, anti-CXCR3, and antiCXCR-4 (also designated anti-fusin) were from Santa Cruz Biotechnology (Santa Cruz, CA). The Santa Cruz goat antibodies were prepared with the following peptides: LERVSSTSPSTGEHELSAGF (CCR1), KSIGRAPEASLQDKEGA (CCR2A), DGVTSTNTPSTGEQEVSAGL (CCR2B), LERTSSVSPSTAEPELSIVF (CCR3), DTPSSSYTQSTMDHDLHDAL (CCR4), ERASSVYTRSTGEQEISVGL (CCR5), SSSRRDSSWSETSEASYSGL (CXCR3), and ALTSVSRGSSLKILSKG (CXCR4). CCR2A staining of the coronary vessels was inhibited with the peptide KSIGRAPEASLQDKEGA.

The antibodies produced by R\&D Systems and LeukoSite were obtained through the National Institutes of Health (NIH) AIDS Research and Reference Reagent Program.

\section{Immunofluorescence and Immunocytochemistry}

The 30,000 cells were grown to confluence for 4 days on gelatin-coated, glutaraldehyde crosslinked coverslips (34). After fixation for $30 \mathrm{~min}$ with $3 \%$ paraformaldehyde at $4^{\circ} \mathrm{C}$, the cells were permeabilized with $0.1 \%$ Triton, incubated for 1 hr with a primary antibody (MAb or PAb at 5 
$\mu \mathrm{g} / \mathrm{ml}$ ), washed, and then incubated with FITCconjugated secondary antibody. For dual staining, two primary mouse and rabbit antibodies and two secondary FITC- or Texas Red-conjugated antibodies specific for mouse or goat immunoglobulin G (IgG) were used. Immunofluorescent preparations were viewed using either an Olympus Research microscope with a mercury lamp or a Zeiss LSM 310 confocal laser microscope. Immunocytochemical staining of the endothelia was done using the DAKO LSAB + Peroxidase kit (DAKO) as described previously (35).

\section{Flow-Cytometric Analysis of Chemokine Receptors}

The endothelial cells were removed from the flask using the Cell Dissociation Buffer EnzymeFree (Life Technologies, Gaithersburg, MD). All staining steps were performed on ice. The endothelial cells were washed with phosphate-buffered saline (PBS)/1\% bovine serum albumin (BSA) by centrifugation. The cells were then resuspended in PBS/1\% BSA and stained with 2 $\mu \mathrm{g} / \mathrm{ml}$ of each primary antibody (CXCR4 MAb, CCR5 MAb, CXCR4 MAb, or IgG isotype control) for $30 \mathrm{~min}$. After two washes with PBS/1\% BSA, rabbit anti-mouse FITC-antibody was added for $30 \mathrm{~min}$. The cells were washed two times and resuspended in PBS/1\% BSA. Finally, the cells were fixed with $2 \%$ paraformaldehyde. Receptor expression on the endothelia was analyzed by EPICS XL-MCO (Coulter).

\section{Histochemistry}

Fresh myocardium from an explanted heart of two patients with dilated cardiomyopathy was obtained from the Surgical Pathology Division of the UCLA Department of Pathology. Specimens were embedded in O.C.T. embedding medium from Sakura Finetek U.S.A. (Torrance CA) and snap frozen in liquid nitrogen and dry ice. Cryostat sections were fixed in $4 \%$ paraformaldehyde in $0.1 \mathrm{M}$ PBS for $30 \mathrm{~min}$ at $4^{\circ} \mathrm{C}$. After washing in PBS the slides were incubated with blocking buffer containing $0.5 \% \mathrm{BSA}$ and then with the primary antibody diluted 1:200 in PBS with $0.1 \%$ Triton X-100 (Sigma) for $12-18 \mathrm{hr}$ at $4^{\circ} \mathrm{C}$. As the primary antibody, mouse $M A b$ to CCR3, CXCR4, or CCR5 (RED), or goat PAb to CCR1, CCR2A, CCR2B, CCR3, CCR4, or CCR5 (Santa Cruz Biotechnology) was used. The slides were washed in PBS containing Tween 20 (PBST) (Sigma) for $5 \mathrm{~min}$ and incubated sequentially with biotinylated rabbit anti-mouse and biotinylated swine anti-rabbit antisera (both from DAKO) for $30 \mathrm{~min}$ at room temperature. Slides were then washed in PBST and incubated with streptavidin-horseradish peroxidase (DAKO) for $30 \mathrm{~min}$. Following the diaminobenzidine reaction, the slides were counterstained with hematoxylin. In the case of the goat PAb, the second step consisted of biotin-conjugated anti-goat antibody. This was followed by avidin and biotinylated horseradish peroxidase (ABC Staining System, Santa Cruz Biotechnology).

\section{Endothelial Chemotaxis Assay}

Chemotaxis of endothelia by CC chemokines was tested in 96-well chemotaxis chambers (NeuroProbe, Gaithersburg, MD), and $50 \mu \mathrm{l}$ of EGM $^{\mathrm{R}}$-2-MV Bullet Kit medium (Clonetics/BioWhittaker) with RANTES, MIP- $1 \alpha$, or MIP- $1 \beta$ at the indicated concentration was placed in the lower chamber and 1000 endothelial cells in 20 $\mu \mathrm{l}$ medium was introduced in the upper well above the plate (with $8-\mu \mathrm{m}$ pores) separating the chambers. The chamber was incubated at $37^{\circ} \mathrm{C}$ for $48 \mathrm{hr}$, then the plate was removed and the cells on the upper surface were removed using a scraper. The lower surface of the plate was stained using $0.1 \%$ crystal violet and the cells were enumerated using $100 \times$ magnification in a horizontal strip across each well. The number of cells was multiplied by 4 to obtain the total number of transmigrated cells.

\section{Results}

Coronary and Brain Endothelia Display CCR3, CXCR4, and CCR5 In Vitro

We investigated the expression of chemokine receptors in cultured endothelia using immunofluorescence and immunoperoxidase techniques with monoclonal antibody (MAb) (CXCRl, CXCR2, CXCR4, CCR3, and CCR5) and/or polyclonal antibody (PAb) (CXCR3, CXCR4, CCR1, CCR2A, CCR2B, CCR3, CCR4, and CCR5). The specificity of each reaction was shown by the absence of staining when the primary antibody to a chemokine receptor was omitted or substituted with an isotype-matched IgG (Fig. 1J-L). CAEC and BMVEC showed strongly positive staining by the immunofluorescence (Fig. 1A-I) and the immunoperoxidase technique (not shown) with antiCCR3, -CXCR4, and -CCR5. The staining of $B M V E C$ and CAEC was consistently greater when the cells were fixed before the staining than when 

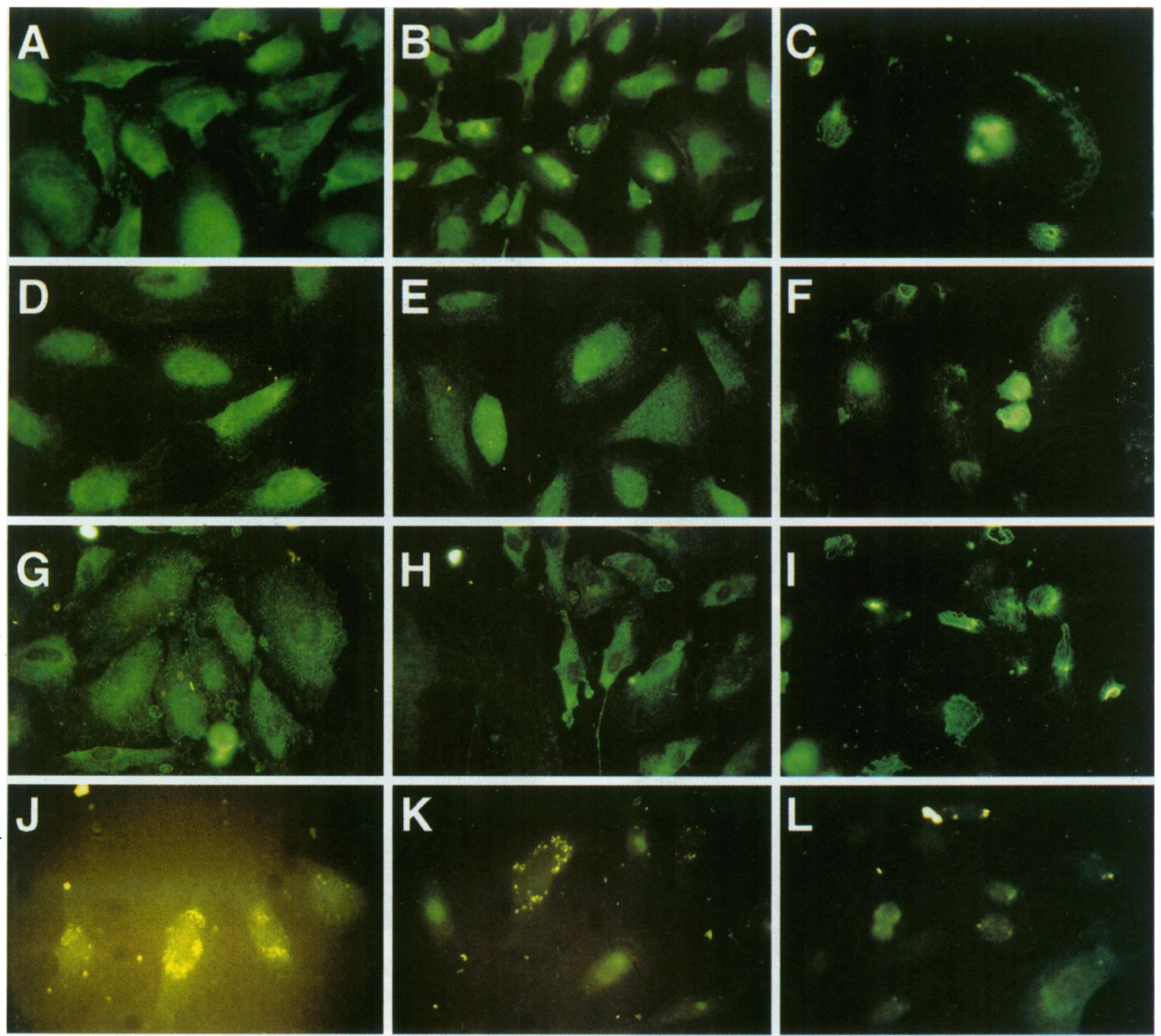

Fig. 1. Immunofluorescent staining of CCR3, CCR5, and CXCR4 chemokine receptors in BMVEC and CAEC. Human brain (A, D, G, J) and coronary (B, C, E, F, H, I, K, L) endothelia were grown on coverslips coated with glutaraldehydefixed gelatin (34). Confluent monolayers were stained by the immunofluorescent technique using MAb against CCR3 (IgG2A isotype) (A-C), CCR5 (IgG2B isotype) (D-F), CXCR4 (IgG2A isotype) (G-

they were stained without fixation (compare Fig. 1B with Fig. 1C, Fig. 1E with Fig. IF, and Fig. $1 \mathrm{H}$ with Fig. 1I). In addition, two CC chemokine receptors (CCR2A and CCR4) were positively stained by immunocytochemistry in both CAEC and BMVEC, and two CXC chemokine receptors (CXCR1 and CXCR3) were stained in BMVEC but not in CAEC (data not shown). CXCR2 was negative by immunocytochemical staining in CAEC and BMVEC (data not shown).

Laser confocal microscopic examination of
I), or an IgG mouse isotype antibody, IgG2A (J, K), or IgG2B (L). The cells were either permeabilized first with Triton (A, B, D, E, G, H, J, K), or they were stained without permeabilization $(C, F, I, L)$. The IgG isotype-stained samples were photographed using a six times longer exposure (compared to MAb-stained preparations) to demonstrate the background autofluorescence staining of the endothelia.

CAEC revealed CXCR4 (Fig. 2A) and CCR5 (Fig. 2B) (green) in a patchy distribution along the plasma membrane and von Willebrand factor (VWF) (red) in a granular-to-homogeneous pattern in the perinuclear location. In contrast to this pattern seen in CAEC, in BMVEC both CCR5 and CXCR4 were expressed in the cytoplasm and on the plasma membrane. CXCR4 (Fig. 3B) was strongly expressed and showed substantial colocalization with VWF in the cytoplasm. CCR5 expression was weak (Fig. 3A). 

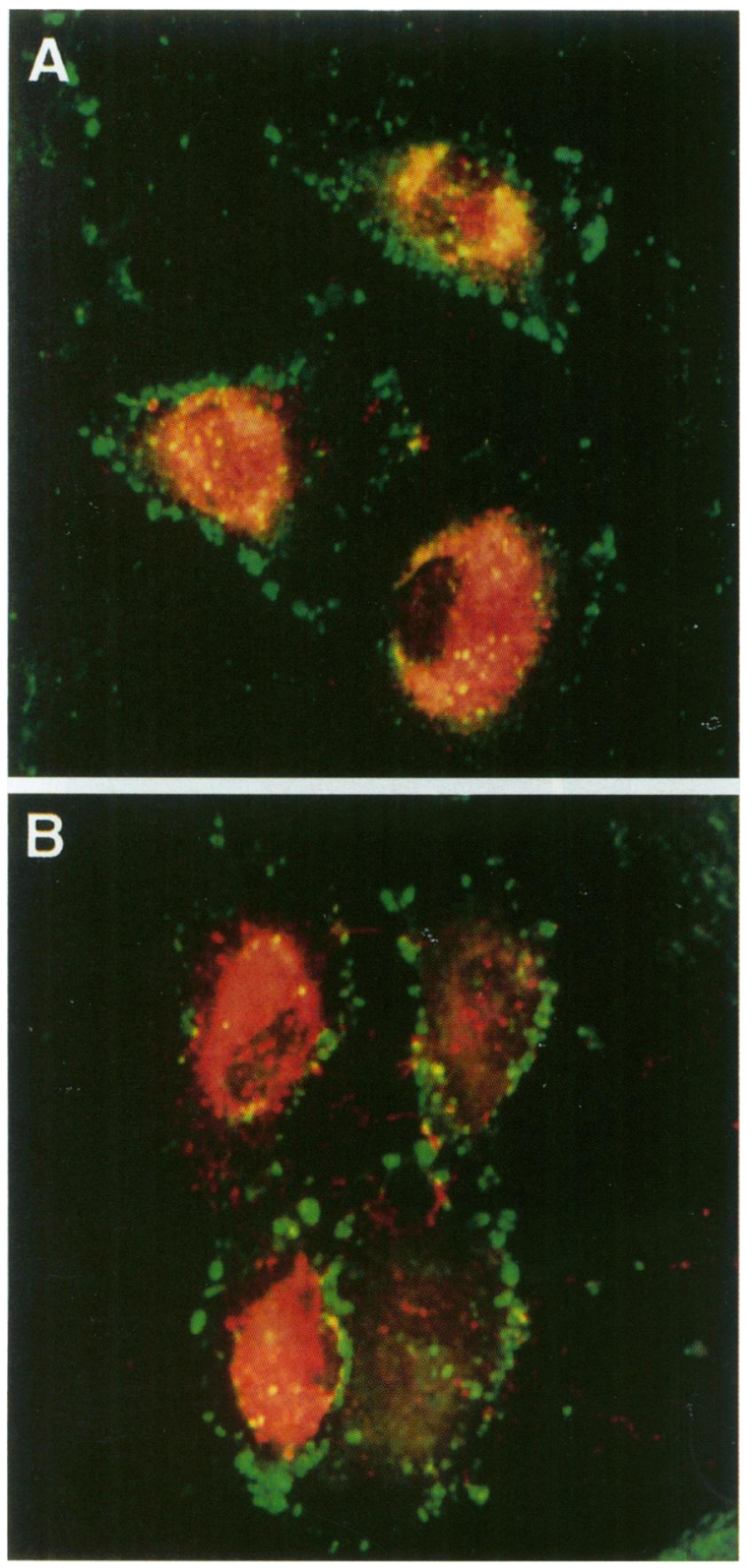

Fig. 2. Confocal microscopy of the subcellular localization of CXCR4 (A) and CCR5 (B) in CAEC. CAEC were grown on coverslips coated with glutaraldehyde-fixed gelatin (34). The cells were double stained using, as the primary antibody, MAb to CXCR4 or CCR5 followed by the rabbit antibody to von Willebrand factor and, as the secondary antibody, mouse or rabbit IgG labeled with FITC or Texas Red, respectively. Chemokine receptors CCR5 and CXCR4 appear as green and von Willebrand factor as red.

CCR3, CXCR4, and CCR5 Receptor Density in CAEC, $B M V E C$, and HUVEC

The expression of chemokine receptors in BMVEC, CAEC, and HUVEC was quantified us-
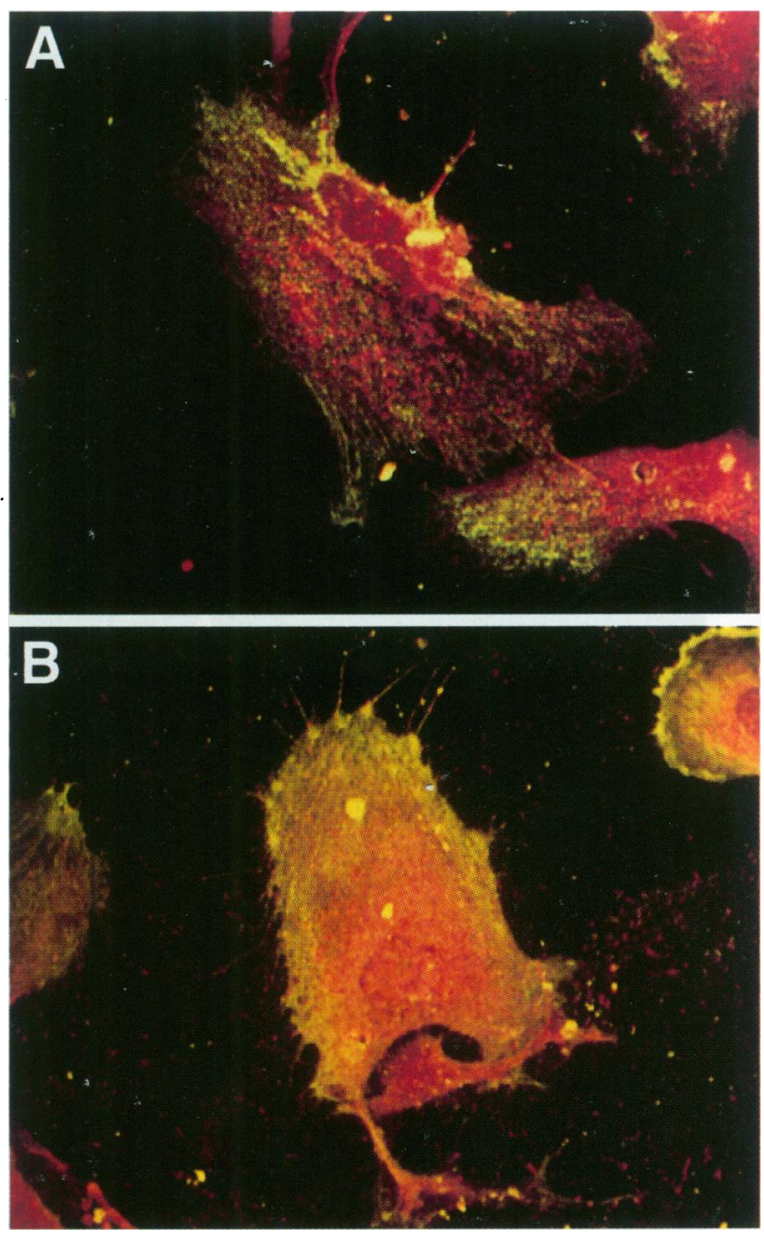

Fig. 3. Confocal microscopy of the subcellular localization of CCR5 (A) and CXCR4 (B) in BMVEC. BMVEC were grown on coverslips and stained as described in Figure 2.

ing flow cytometry performed with the cells stained without prior fixation. In the case of BMVEC, the receptor density (\% staining by the specific antibody - \% staining by the IgG isotype control) was determined in two experiments as follows: CCR3, 45\% and 77\%; CXCR4, $41 \%$ and $51 \%$; and CCR5, $4 \%$ and $5 \%$. In one additional experiment, CXCR4 was $40 \%$ and CCR5 was $27 \%$. In CAEC, the density was measured in two experiments with the following results: CCR3, $11 \%$ and $21 \%$; CXCR4, $41 \%$ and $45 \%$, and CCR $5,6 \%$ and $4 \%$. In HUVEC, the results were $7 \%$ with CCR $3,48 \%$ with CXCR4, and $8 \%$ with CCR5. The flow-cytometric diagrams of a typical experiment illustrate the above receptor densities, as follows: in BMVEC the order was CCR3 = CXCR4 > CCR5 (Fig. 4A); in CAEC the order was CXCR4 > CCR3 > CCR5 (Fig. 4B); and in HUVEC the order was CXCR4 $\gg$ CCR5 and CCR3 


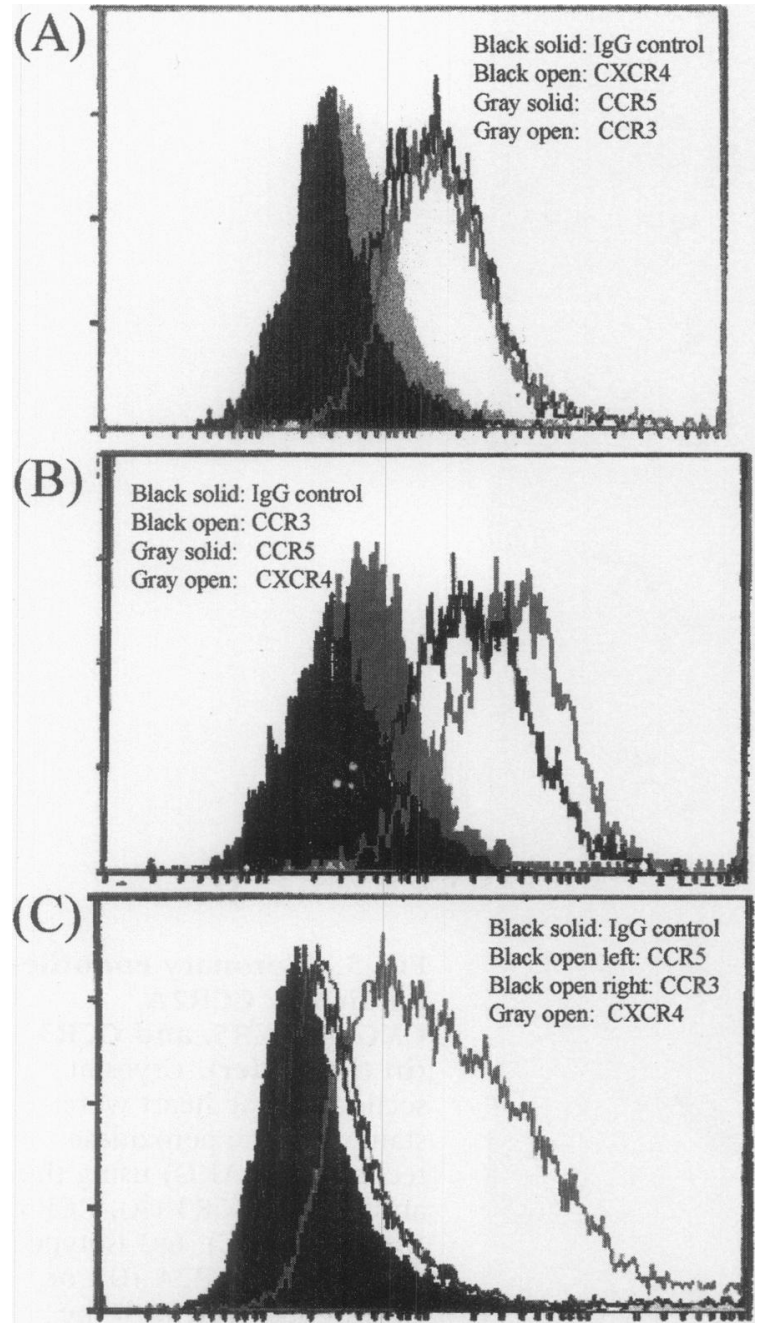

Fig. 4. Chemokine receptor expression in CAEC, BMVEC, and HUVEC evaluated by flow cytometry. Endothelial cells BMVEC (A), CAEC (B), and HUVEC (C) were lifted from the flask in a cell-dissociation buffer, washed, and stained using the monoclonal antibody to CCR3, CCR5, or CXCR4, and analyzed by flow cytometry as described in $\mathrm{Ma}$ terials and Methods.

(Fig. 4C). When the cells were fixed first and stained second, the percentage of staining increased.

CCR3, CXCR4, CCR5, and CCR2A Are Expressed in Coronary Endothelia of the Heart

Immunohistochemical staining of the human heart sections was performed with three heart specimens. Typical results revealed a strong $(4+)$ staining of small venules and capillaries in the myocardium with goat polyclonal antiserum to CCR2A (Fig. 5D), a moderately strong (3+) staining with a monoclonal antibody to CXCR4 (Fig. 5C), a moderate $(2+)$ staining with monoclonal anti-CCR5 (Fig. 5B), and a weak $(1+)$ staining with mouse MAb to CCR3 (Fig. 5A). A positive staining reaction appeared to involve the endothelial cell cytoplasm as well as the cell membrane (Fig. 5C, D). IgG isotype control was negative (Fig. 5F). Preincubation of anti-CCR2A with its blocking peptide completely inhibited the staining reaction (compare Fig. 5D with Fig. 5E). The results of immunostaining of the heart tissues with anti-CCR1, CCR2B, and CCR4 were negative.

RANTES, MIP-1 $\alpha$, and MIP-1 $\beta$ Stimulate Migration of Coronary Endothelia

CAEC were tested for the chemotactic responses to the CC chemokines RANTES, MIP- $1 \alpha$, and MIP-1 $\beta$. Each of these chemokines produced endothelial cell migration at a concentration above $12.5 \mu \mathrm{g} / \mathrm{ml}$ (Fig. 6). A second experiment confirmed the chemotactic responses to these chemokines but the threshold for chemotactic activity was one dilution lower $(12.5 \mu \mathrm{g} / \mathrm{ml})$ with each chemokine.

\section{Discussion}

We have shown that human coronary and brain endothelia express to a different degree the CC chemokine receptors CCR3 and CCR5, and the CXC receptor CXCR4. Whereas in CAEC usually only CXCR4 achieved a high density, in BMVEC, CCR3 and CXCR4 were both highly expressed. CCR5 was expressed at a low density in both endothelia. The effect of successive in vitro passages of the endothelia appeared to decrease the chemokine receptor density. The pattern of a strong expression of CXCR4 and a weak expression of CCR3 was also found in the coronary vessel endothelia in the heart. Two other chemokine receptors, CCR2A and CCR4, were detected by immunostaining in both CAEC and BMVEC, and CXCRI and CXCR3 were found in BMVEC only. In addition, CCR5 and CXCR4 mRNAs were detected in BMVEC by reverse transcriptase polymerase chain reaction (RT-PCR) with five times greater expression of CXCR4 mRNA (C. Gujuluva et al., unpublished results). The presence of multiple chemokine receptors in endothelia is supported by the RT-PCR data of Gupta et al. (20) in HUVEC and BMVEC, which showed an abundant transcription of CXCR4 and a 

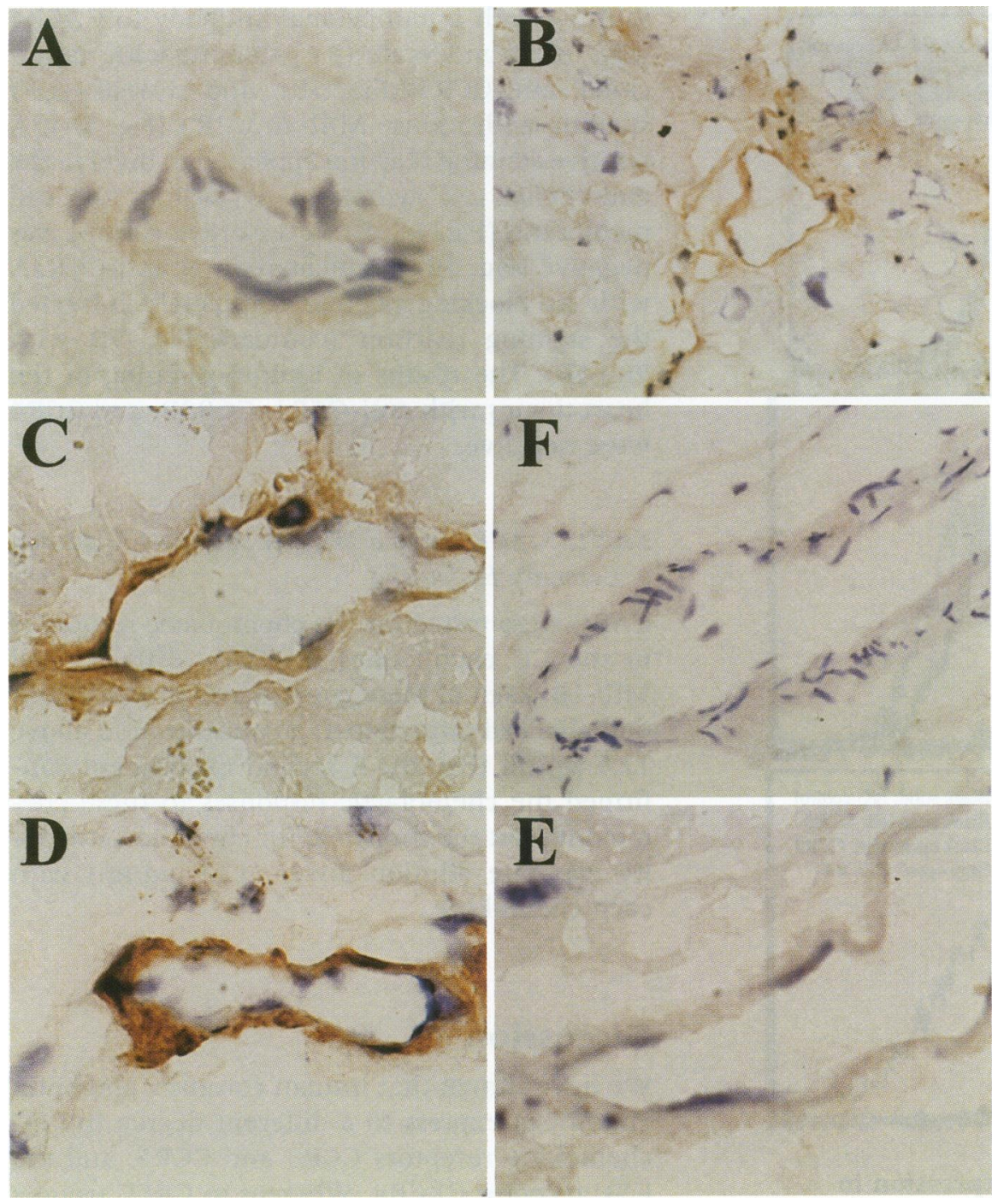

Fig. 5. Coronary endothelia display CCR2A, CXCR4, CCR5, and CCR3 (in this order). Cryostat sections of the heart were stained by the peroxidase technique (DAKO) using the antibody to CCR3 (A), CCR5 (B), CXCR4 (C), IgG isotype control (F), CCR2A (D), or CCR2A absorbed with the peptide KSIGRAPEASLQDKEGA (E). weaker transcription of CXCRl, CCRl, and CCR2. A recent RT-PCR study of HUVEC noted the presence of CXC chemokine mRNAs but not CC chemokine mRNAs (21), and the authors speculated that endothelial cells do not express $\mathrm{CC}$ chemokine receptors. However, their conclusions were based on transcriptional studies and involved only HUVEC. Our flow-cytometric data demonstrate that whereas CXCR4 and CCR3 are both highly expressed in BMVEC, only CXCR4 is highly expressed in CAEC and HUVEC. These results were subjected to quantitative variation, with preservation of these relationships between the receptors, due to the effects of cell passage and fixation. The fixation before staining increased the staining density because of staining of intracellular receptors. These results strongly suggest that brain, coronary, and umbilical endothelia differ from each other with respect to chemokine receptor expression. The histochemical study of the heart provided further evidence of the relevance of chemokine receptors to inflammatory responses of coronary endothelia, as four chemokine receptors, CCR2A, CCR3, CCR5, and $\mathrm{CXCR} 4$, were found on the coronary vessels but only CXCR4 and CCR2A were expressed at a high level, in agreement with the above results.

Chemokine HIV-1 coreceptors, CCR5 and CXCR4, play a crucial role in HIV-1 entry into the blood cells (36). On leukocytes, CXCR4 serves as a coreceptor for $\mathrm{T}$ cell line tropic " $\mathrm{X}$ " strains, whereas the $\mathrm{CC}$ chemokine receptor CCR5 is a main coreceptor for M-tropic " $R$ " strains. In blood-derived dendritic cells, which express CXCR4 and CCR5 mRNAs (37), the entry of M-tropic strains is inhibited by the chemokine RANTES and the entry of the IIIB strain by the chemokine SDF-1. CD $34^{+}$progenitors are 


\section{(A)}



(B)

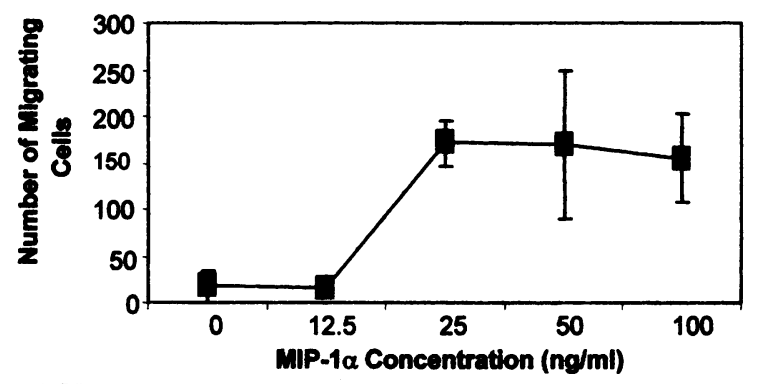

(C)

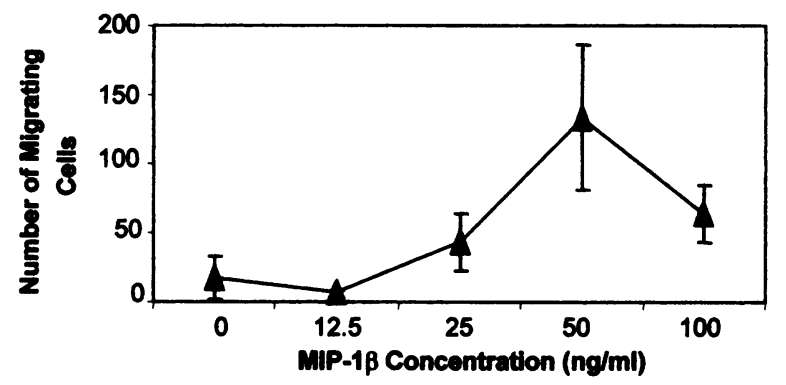

Fig. 6. Endothelial cell migration across a NeuroProbe chamber filter. One thousand CAEC were seeded on top of each well in a NeuroProbe chamber ( $8 \mu \mathrm{m}$ pore diameter) with each tested chemokine, RANTES (A), MIP-l $\alpha$ (B), or MIP-1 $\beta$ $(\mathrm{C})$, at the indicated concentration in the lower chamber. The endothelia were allowed to migrate for $48 \mathrm{hr}$, then the filter was removed and the cells on the top of each well were scraped. After staining by crystal violet, the transmigrated cells on the lower surface of each well were determined by a microscopic count as described in Materials and Methods.

susceptible to infection by various HIV-l strains and cognate ligands for CXCR4 and CCR5 modulate HIV-1 infection (38).

CC and CXC chemokine receptors belong to a large family of cell surface, seven-transmembrane domain, $G$ protein-coupled receptors that bind and signal upon binding of one or more CC or CXC chemokines (3). CCR2 is a receptor previously detected in monocytes, dendritic cells, natural killer (NK) cells, and T lymphocytes, but not in neutrophils, and is thus considered to be important in chronic inflammation, including atherosclerosis. CCR2 has recently been shown to function as a receptor for MCP-1 on endothelia, inducing their chemotactic migration and possibly endothelial wound repair (39). CCR3 is found in brain microglia, in which it mediates HIV-l entry (40). CCR5 is a major HIV-1 coreceptor, which plays a crucial role in HIV infection of peripheral blood-derived dendritic cells, $\mathrm{CD} 4^{+}$hematopoeitic progenitor cells, and activated/memory Thl lymphocytes $(41,42)$. CCR5 expression on freshly isolated $\mathrm{T}$ cells is increased by activation with IL-2 and other stimuli (41). In contrast to the pivotal significance of CCR5 on blood cells for HIV-1 infection (as well as other infections), the role of CCR5 in neurons, astrocytes, and other cells has not been established. CXCR4 is the major chemokine coreceptor for T-tropic HIV-l strains that is widely distributed on most hematopoietic cell types, endothelial cells (20), neurons in both the peripheral and central nervous systems (43), microglia, and astrocytes (40). CXCR4 appears to play an essential role in development, as shown in mice (22).

Our studies of the heart tissues showed that CCR2A and CXCR4, and, to a lesser degree, CCR3 and CCR5 were displayed on coronary endothelia. We have not excluded a possibility that this staining pattern is related to the health status of each donor, as the hearts were removed before transplantation of patients with congestive cardiomyopathy. The receptors were distributed diffusely in the endothelial cytoplasm and on the plasma membrane, in agreement with the confocal microscopic results in coronary endothelia. The receptors were not limited to the abluminal side of endothelia, as found by Andjelkovic et al. (18), by staining isolated microvessels with MAb to CCR1, CCR3, and CCR5.

The functional role of chemokine receptors on brain endothelia, in particular CCR3, CXCR4, and, to a lesser degree, CCR5, could be significant for HIV-1 neuroinvasion. Macrophagetropic strains circulating at a very high titer $\left(10^{7}\right.$ RNA copies/ml plasma) during the primary infection could find early entry into the brain because of a high expression on BMVEC of CCR3 and a variable CCR5 expression. Our recent work strongly suggests that HIV invades across brain endothelia via both the paracellular $(30,31)$ and the transcellular route (C. Gujuluva et al., unpublished results) without undergoing replication in BMVEC. However, we have not 
yet obtained conclusive evidence for the role of chemokine receptors in viral transcytosis. After the neuroinvasion, the macrophage-tropic HIV-1 strains utilizing CCR 3 and CCR5 could effectively enter the microglia via CCR3 (40) and CCR5 (44). SIV infects simian brain endothelial cells by a CCR5-dependent mechanism, which is inhibited by the CCR5 ligand RANTES (45), but this route may be limited in human brain endothelia because of the lower expression of CCR5. The presence of CXCR4 on brain endothelia may allow the T-tropic strains emerging late in the disease to infect the brain. Coronary endothelia, unlike brain endothelia, appear to have a reduced expression of CCR3, which may limit the ability of macrophage-tropic strains circulating early after infection to infect the heart. The coronary endothelia, however, express CXCR4 and may be susceptible to T-tropic strains that emerge late in the course of AIDS, when HIV-1 cardiomyopathy is apt to develop.

\section{Acknowledgments}

This work was supported in part by NIH grant DA10442 and a subcontract NS 26126 to M.F. and M.C.G., HL 48493 to M.H.W., and RO-1 NS 26310 and RO-1 HL 61951 to K.-S.K.A.R.B. is supported in part by grants from NIH HL-42550, The Methodist Hospital Foundation, a Chao Fellowship, and ALA RG 068. The authors acknowledge the UCSD Center for AIDS Research and NIH DAIDS 2 P30 AI36214-07. We thank Philip Murphy for incisive comments, Matthew Schibler for assistance with confocal microscopy, and Carol Appleton for technical assistance with illustrations.

\section{References}

1. Luster A. (1998) Chemokines-chemotactic cytokines that mediate inflammation. N. Engl. J. Med. 338: 436-445.

2. Taub D. (1996) Chemokine-leukocyte interactions-the voodoo that they do so well. Cytokine Growth Factors Rev. 7: 355-376.

3. Murphy P. (1996) Chemokine receptors: structure, function and role in microbial pathogenesis. Cytokine Growth Factor Rev. 7: 47-64.

4. Berliner JA, Territo MC, Sevanian A, et al. (1990) Minimally modified low density lipoprotein stimulates monocyte endothelial interactions. J. Clin. Invest. 85: 1260-1266.

5. Maat-Schieman ML, van Duinen SG, Rozemuller AJ, Haan J, Roos RA. (1997) Association of vas- cular amyloid beta and cells of the mononuclear phagocyte system in hereditary cerebral hemorrhage with amyloidosis (Dutch) and Alzheimer disease. J. Neuropathol. Exp. Neurol. 56: 273-284.

6. Sorensen TL, Ransohoff RM (1998) Etiology and pathogenesis of multiple sclerosis. Semin. Neurol. 18: $287-294$.

7. Glass JD, Fedor H, Wesselingh SL, McArthur JC. (1995) Immunocytochemical quantitation of human immunodeficiency virus in the brain: correlations with dementia. Ann. Neurol. 38: 755-762.

8. Navab M, Imes SS, Hama SY, et al. (1991) Monocyte transmigration induced by modification of low density lipoprotein in cocultures of human aortic wall cells is due to induction of monocyte chemotactic protein 1 synthesis and is abolished by high density lipoprotein. J. Clin. Invest. 88: 2039-2046.

9. Sorensen TL, Tani M, Jensen J, et al. (1999) Expression of specific chemokines and chemokine receptors in the central nervous system of multiple sclerosis patients. J. Clin. Invest. 103: 807-815.

10. Boisvert WA, Santiago R, Curtiss LK, Terkeltaub RA. (1998) A leukocyte homologue of the IL-8 receptor CXCR-2 mediates the accumulation of macrophages in atherosclerotic lesions of LDL receptor-deficient mice. J. Clin. Invest. 101: 353-363.

11. Ross R. (1999) Atherosclerosis-an inflammatory disorder. N. Engl. J. Med. 340: 115-126.

12. Terkeltaub R, Boisvert WA, Curtiss LK. (1998) Chemokines and atherosclerosis. Curr. Opin. Lipidol. 9: 397-405.

13. Schmidtmayerova H, Nottet H, Nuovo G. (1996) HIV-1 infection alters chemokine beta-peptide expression in human monocytes: implications for recruitment of leukocytes into brain and lymph nodes. Proc. Soc. Natl. Acad. Sci. U.S.A. 93: 700-704.

14. Richmond A, Mueller S, White JR, Schraw W. (1997) C-X-C chemokine receptor desensitization mediated through ligand-enhanced receptor phosphorylation on serine residues. Methods Enzymol. 288: 3 .

15. Hub E, Rot A. (1998) Binding of RANTES, MCP-1, MCP-3, and MIP- $1 \alpha$ to cells in human skin. Am. J. Pathol. 152: 749-757.

16. Tanaka Y, Adams DH, Shaw S. (1993) Proteoglycans on endothelial cells present adhesion-inducing cytokines to leukocytes. Immunol. Today 14: 111-115.

17. Luster $A D$, Greenberg SM, Leder P. (1995) The IP-10 chemokine binds to a specific cell surface heparan sulfate site shared with platelet factor 4 and inhibits endothelial cell proliferation. J. Exp. Med. 182: 219-231.

18. Andjelkovic AV, Spencer DD, Pachter JS. (1999) Visualization of chemokine binding sites on human brain microvessels. J. Cell Biol. 145: 403-412.

19. Volin M, Joseph L, Shockley M, Davies P. (1998) Chemokine receptor CXCR4 expression in endo- 
thelium. Biochem. Biophys. Res. Commun. 242: 4653.

20. Gupta S, Lysko P, Pillarisetti K, Ohlstein E, Stadel J. (1998) Chemokine receptors in human endothelial cells. J. Biol. Chem. 273: 4282-4287.

21. Feil C, Augustin HG. (1998) Endothelial cells differentially express functional CXC-chemokine receptor-4 (CXCR-4/fusin) under the control of autocrine activity and exogenous cytokines. Biochem. Biophys. Res. Commun. 247: 38-45.

22. Tachibana $K$, Hirota S, Iizasa H, et al. (1998) The chemokine receptor CXCR4 is essential for vascularization of the gastrointestinal tract [see comments]. Nature 393: 591-594.

23. Nagasawa T, Hirota S, Tachibana $K$, et al. (1996) Defects of B-cell lymphopoiesis and bone-marrow myelopoiesis in mice lacking the $\mathrm{CXC}$ chemokine PBSF/SDF-1. Nature 382: 635-638.

24. Strieter RM, Polverini PJ, Kunkel SL, et al. (1995) The functional role of the ELR motif in CXC chemokine-mediated angiogenesis. J. Biol. Chem. 270: 27348-27357.

25. Page C, Rose M, Yacoub M, Pigott R. (1992) Antigenic heterogeneity of vascular endothelium. Am. J. Pathol. 141: 763-683.

26. Gerritsen M. (1987) Functional heterogeneity of vascular endothelial cells. Biochem. Pharmacol. 36: 2701-2711.

27. Craig L, Spelman J, Strandberg J, Zink M. (1998) Endothelial cells from diverse tissues exhibit differences in growth and morphology. Microvasc. Res. 55: 65-76.

28. Vicenzi E, Biswas P, Mengozzi M, Poli G. (1997) Role of pro-inflammatory cytokines and beta-chemokines in controlling HIV replication. J. Leukocyte Biol. 62: 34-40.

29. Huang L, Bosch I, Hofmann W, Sodroski J, Pardee A. (1998) Tat protein induces HIV-1 coreceptors and promotes infection with both macrophagetropic and T-lymphotropic HIV-1 strains. J. Virol. 72: 8952-8960.

30. Fiala M, Looney DJ, Stins $M$, et al. (1997) TNFalpha opens a paracellular route for HIV-1 invasion across the blood-brain barrier. Mol. Med. 3: 553-564.

31. Zhang L, Looney D, Taub D, et al. (1998) Cocaine opens the blood-brain barrier to HIV-1 invasion. J. Neurovirol. 4: 619-626.

32. Stins M, Gilles F, Kim K. (1997) Selective expression of adhesion molecules on human brain microvascular endothelial cells. J. Neuroimmunol. 76: 81-90.

33. Huang AJ, Furie MB, Nicholson SC, Fischbarg J, Liebovitch LS, Silverstein SC. (1988) Effects of human neutrophil chemotaxis across human en- dothelial cell monolayers on the permeability of these monolayers to ions and macromolecules. J. Cell. Physiol. 135: 355-366.

34. Burns AR, Walker DC, Brown ES. (1997) Neutrophil transendothelial migration is independent of tight junctions and occurs preferentially at tricellular corners. J. Immunol. 159: 2893-2903.

35. Fiala M, Rhodes RH, Shapshak P, et al. (1996) Regulation of HIV-1 infection in astrocytes: expression of Nef, TNF- $\alpha$ and IL- 6 is enhanced in coculture of astrocytes with macrophages. $\mathrm{J}$. Neurovirol. 2: 158-166.

36. Berger E. (1998) Introduction: HIV co-receptors solve old questions and raise many new ones. Immunology 10: 165-168.

37. Granelli-Piperno A, Moser B, Pope $M$, et al. (1996) Efficient interaction of HIV-1 with purified dendritic cells via multiple chemokine receptors. J. Exp. Med. 184: 2433-2438.

38. Ruiz M, Cicala C, Arthos J, et al. (1998) Peripheral blood-deriverd CD34+ progenitor cells: CXC chemokine receptor 4 and $\mathrm{CC}$ chemokine receptor 5 expression and infection by HIV. J. Immunol. 161: 4169-4176.

39. Weber KS, Nelson PJ, Grone HJ, Weber C. (1999) Expression of CCR2 by endothelial cells: implications for MCP-1 mediated wound injury repair and In vivo inflammatory activation of endothelium. Arterioscler. Thromb. Vasc. Biol. 19: 2085-2093.

40. He J, Chen Y, Farzan M, et al. (1997) CCR3 and CCR5 are co-receptors for HIV-1 infection of microglia. Nature 385: 645-649.

41. Bleul CC, Wu L, Hoxie JA, Springer TA, Mackay CR. (1997) The HIV coreceptors CXCR4 and CCR5 are differentially expressed and regulated on human T lymphocytes [see comments]. Proc. Natl. Acad. Sci. U.S.A. 94: 1925-1930.

42. Loetscher $P$, Uguccioni M, Bordoli L, et al. (1998) CCR5 is characteristic of Thl lymphocytes [letter]. Nature 391: 344-345.

43. Hesselgesser J, Halks-Miller M, DelVecchio V, et al. (1997) CD4-independent association between HIV-1 gpl20 and CXCR4: functional chemokine receptors are expressed in human neurons. Curr. Biol. 7: 112-121.

44. Albright A, Shieh JT, Itoh T, et al. (1999) Microglia express CCR5, CXCR4, and CCR3, but of these, CCR5 is the principal coreceptor for HIV-1 dementia isolates. J. Virol. 73: 205-223.

45. Edinger AL, Mankowski JL, Doranz BJ, et al. (1997) CD4-independent, CCR5-dependent infection of brain capillary endothelial cells by a neurovirulent simian immunodeficiency virus strain. Proc. Natl. Acad. Sci. U.S.A. 94: 14742-14747. 